\title{
THE LOW ENERGY DIFFUSE COSMIC X-RADIATION
}

\author{
B. G. WILSON and A. J. BAXTER \\ Dept. of Physics, The University of Calgary, Calgary, Canada
}

\begin{abstract}
Recent measurements of the low energy diffuse cosmic X-radiation are reported. The results indicate that the large flux observed below $1 \mathrm{keV}$ is inconsistent with an extrapolation of the power law spectrum observed above $2 \mathrm{keV}$. While the radiation between 2 and $10 \mathrm{keV}$ appears largely isotropic, at least to the limits of resolution of the detector, the $0.27 \mathrm{keV}$ radiation shows significant asymmetry with respect to galactic latitude.
\end{abstract}

We report here some of the results obtained from detectors flown in northern Canada during 1968. On one flight, using a Bristol Aerospace Black Brant IV vehicle, a 5-mil beryllium-windowed proportional counter reached an altitude of $880 \mathrm{~km}$ above Churchill, Manitoba, on April 5. The combination of large coning angle and 6-sec spin rate enabled a survey of almost all the northern sky to be made. The $38-\mathrm{cm}^{2}$ detector was covered by a hexagonal aluminum honeycomb limiting the field of view to 0.117 sterad. A $\frac{1}{4}$-mil mylar-windowed counter was flown over Resolute Bay, N.W.T., on October 7 by a Black Brant III to a height of $155 \mathrm{~km}$. This detector was $30 \mathrm{~cm}^{2}$ in area and contained $90 \%$ Argon- $10 \%$ methane gas, with an identical field of view. Due to the peculiar motion of the freely spinning rocket, with the spin period almost exactly twice the coning period, the counter viewed the same quite narrow bands of the sky in successive rotations.

Detailed counter performance and the method of data reduction will be reported elsewhere. In a recent paper (Baxter et al., 1969) we have provided a preliminary spectral analysis of the $0.20-4.0 \mathrm{keV}$ radiation indicating a sharp increase in flux below $1 \mathrm{keV}$. In that paper the error bars shown are purely statistical. Further analysis, taking into account uncertainties in window thickness and calibration, provides the data in Figure 1. Although the efficiency of the mylar window is extremely low between 0.27 and $0.8 \mathrm{keV}$, photons were observed in this energy range, after full consideration of resolution effects. The experimental data appear quite consistent with a smooth increase in flux to the lowest energies observed.

In the $2-10 \mathrm{keV}$ energy range the diffuse radiation is usually quoted as isotropic to within 10\%'. The long flight on April 5 enabled a rather complete analysis of directional intensity to be made (Figure 2). Apart from the obvious contributions of the Crab and Cygnus sources, the sky appears to be rather even in X-ray brightness, to the resolution limit of the collimator (approximately $10^{\circ}$ half-angle). Smaller scale irregularities would not be readily detectable. There is, however, some suggestion that the average intensity above $60^{\circ} \mathrm{N}$ galactic latitude is greater than at lower latitudes.

When the carbon-K transmission band intensities are plotted, however, there is clear evidence of asymmetrical distribution with an approximately 7:1 intensity ratio between directions near the galactic pole and those towards the plane, taking the lower value of the two transit passes as more likely to be uncontaminated by sources of radiation (Figure 3 ). 


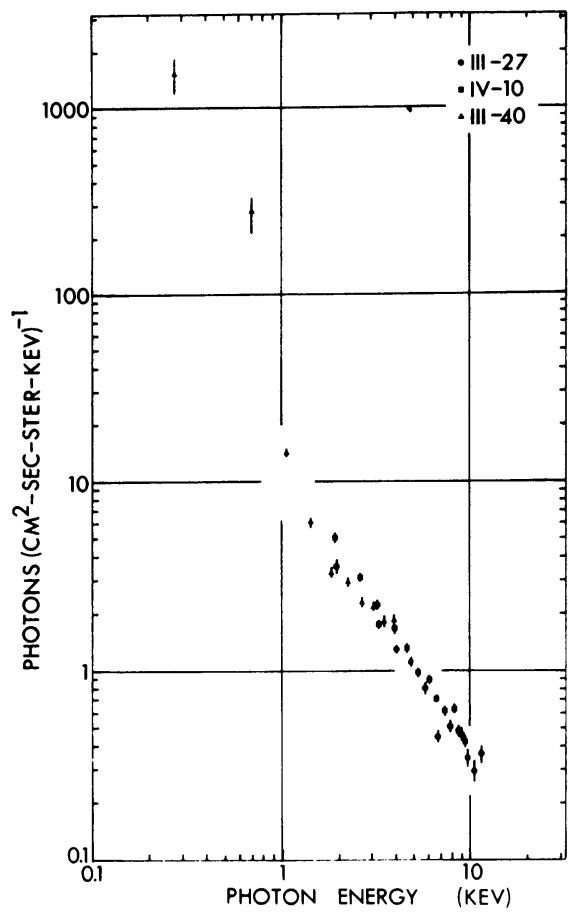

Fig. 1. Spectral results for the intensity of the diffuse X-ray component measured by The University of Calgary series of experiments.

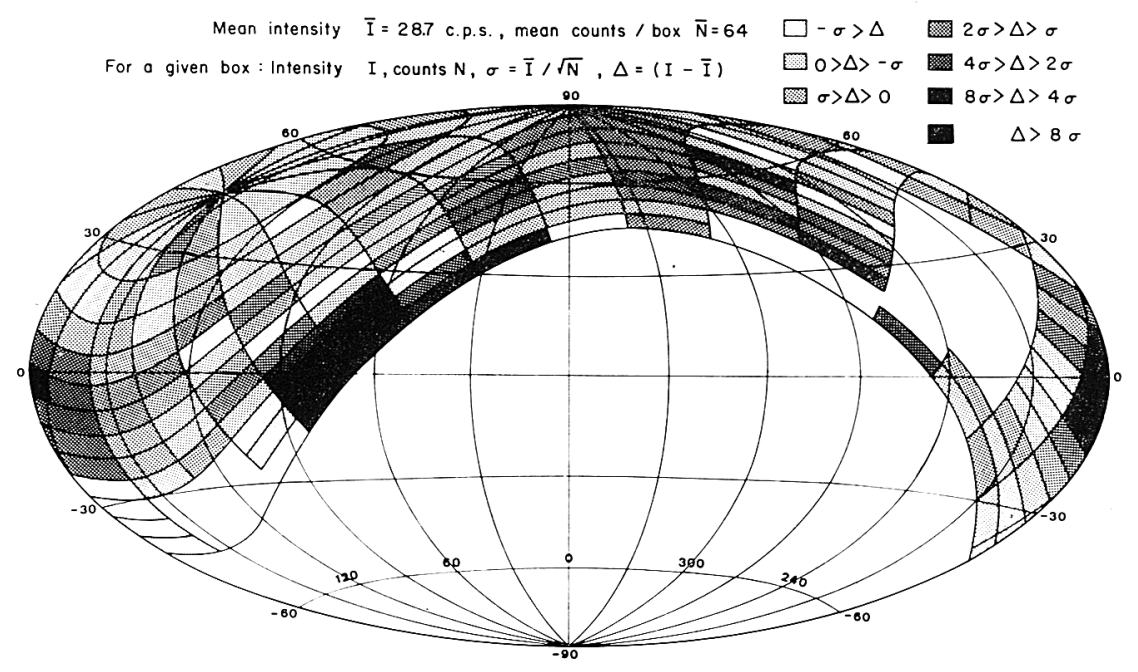

Fig. 2. Distribution of counts in horizon coordinates in the energy range $1.6-10 \mathrm{keV}(5 \mathrm{mil} \mathrm{Be}$, IV-10), superimposed on galactic coordinates $\left({ }^{\mathrm{II}}, b^{\mathrm{II}}, 1950\right)$. 


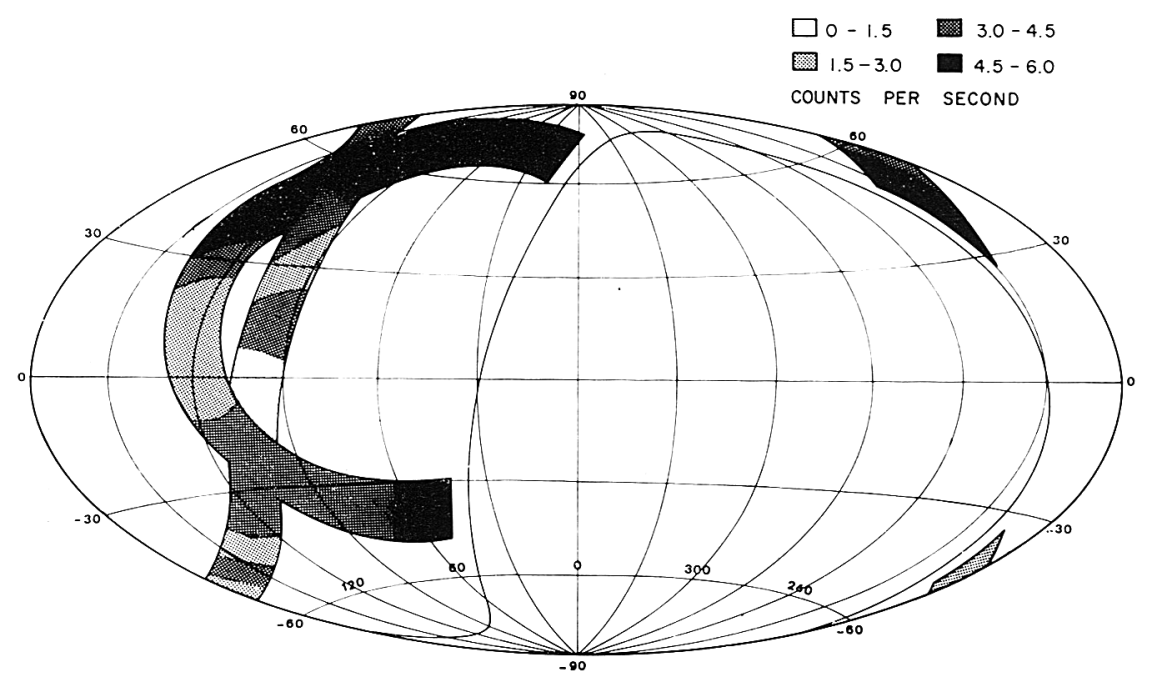

Fig. 3. Distribution in galactic coordinates $\left(l^{\mathrm{II}}, b^{\mathrm{II}}, 1950\right)$ of counts observed in the carbon $\mathrm{K}$ transmission band for the mylar counter (III-40), corrected for detector background.

During this flight the sun was about $20^{\circ}$ below the rocket horizon. Some solar $\mathrm{X}$-ray contributions were noted in the gross counting rate when the detector faced that direction during alternate rotations of the nose cone. No such contribution could be detected for the remaining $95 \%$ of the motion. This solar contribution was not detectable at energies below about $1 \mathrm{keV}$. The X-ray detector was enclosed by a scintillation-veto counter. The effectiveness of this combination in reducing local background effects was evident in the 15:1 reduction in intensity as the detector scanned the earth during each rotation of the nose cone. No horizon effects were noted in the data, suggesting that the observed results are unlikely to be contaminated by soft election precipitation in the polar sky. This conclusion is supported by the lack of anomalous results in a separate thin windowed Geiger Counter as well as by the consistent level of intensity observed by the proportional counter in 11 successive sky scans. The $2.0-4.0 \mathrm{keV}$ results are also in excellent agreement with a wealth of experimental data in this part of the spectrum. Finally, the measured atmospheric absorption of the observed radiation is in good agreement with the predicted attenuation along the line of sight of the detector. (The $1350 \AA$ aluminum coating of the mylar window makes the counter insensitive to ultra violet radiation.) We find it difficult therefore to resist concluding that the measured data are in fact due to cosmic X-rays which exhibit a surprising intensity below $1 \mathrm{keV}$ and considerable anisotropy related to the gross galactic structure.

\section{Reference}

Baxter, A. J., Wilson, B. G., and Green, D. W.: 1969, Astrophys. J. Letters 155, L145. 